DO-TH 07/01

LPHE/07-01

\title{
Photon polarization from helicity suppression in radiative decays of polarized $\Lambda_{b}$ to spin-3/2 baryons
}

\author{
G. Hiller ${ }^{\mathrm{a}}$, M. Knecht ${ }^{\mathrm{b}}$, F. Legger ${ }^{\mathrm{b}, 1}$, and T. Schietinger ${ }^{\mathrm{c}}$ \\ a Institut für Physik, Universität Dortmund, D-44221 Dortmund, Germany \\ ${ }^{\mathrm{b}}$ Laboratory for High-Energy Physics, Ecole Polytechnique Fédérale, \\ CH-1015 Lausanne, Switzerland \\ ${ }^{\mathrm{c}}$ Paul Scherrer Institut, CH-5232 Villigen PSI, Switzerland
}

\begin{abstract}
We give a general parameterization of the $\Lambda_{b} \rightarrow \Lambda(1520) \gamma$ decay amplitude, applicable to any strange isosinglet spin- $3 / 2$ baryon, and calculate the branching fraction and helicity amplitudes. Large-energy form factor relations are worked out, and it is shown that the helicity-3/2 amplitudes vanish at lowest order in soft-collinear effective theory (SCET). The suppression can be tested experimentally at the LHC and elsewhere, thus providing a benchmark for SCET. We apply the results to assess the experimental reach for a possible wrong-helicity $b \rightarrow s \gamma$ dipole coupling in $\Lambda_{b} \rightarrow \Lambda(1520) \gamma \rightarrow p K \gamma$ decays. Furthermore we revisit $\Lambda_{b}$-polarization at hadron colliders and update the prediction from heavy-quark effective theory. Opportunities associated with $b \rightarrow d \gamma$ afforded by high-statistics $\Lambda_{b}$ samples are briefly discussed in the general context of $\mathrm{CP}$ and flavour violation.
\end{abstract}

Key words: Effective theory, B-physics, Baryon polarization and decay PACS: 11.30.Er, 13.30.-a, 13.88.+e, 14.20.Mr

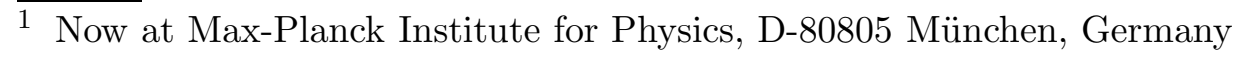

Preprint submitted to Elsevier Science

29 October 2018 


\section{Introduction}

Flavour-changing neutral current decays of the $b$-quark, such as $b \rightarrow s \gamma$ and $b \rightarrow d \gamma$, are important probes of the flavour sector of the theory [1]. While the rates of e.g. $B \rightarrow X_{s} \gamma$ and $B \rightarrow K^{*} \gamma$ are in agreement with the Standard Model (SM), the handedness of the underlying electromagnetic dipole transition $b \rightarrow s \gamma$, which in the SM requires predominantly left-polarized photons $[2]$, is poorly constrained to date [3].

Radiative decays of $b$-flavoured baryons allow the study of spin correlations, giving information on the chirality of the dipole transition. The decay $\Lambda_{b} \rightarrow$ $\Lambda(1116) \gamma$ with subsequent dominant $\Lambda(1116)$ decay to $p \pi^{-}$has been identified as a useful mode to test the SM at colliders $[4,5,6]$. In particular, the spin of the $\Lambda(1116)$ is self-analyzed by its decay, i.e., correlated with the direction of the momentum of the proton. A second spin-asymmetry can be formed if the $\Lambda_{b}$ 's are polarized [5]. The main drawback of experimental studies involving $\Lambda(1116)$, however, is its weak decay: Due to the associated long decay length, a large fraction of these baryons decays outside the inner (vertex) part of a given detector, posing formidable difficulties for the decay reconstruction [7].

For this reason, it was suggested in Ref. [7] to study $b \rightarrow s \gamma$-mediated $\Lambda_{b^{-}}$ decays to heavier $\Lambda$-baryons (resonances), which decay strongly and copiously into $N \bar{K}$ [8]. The self-analyzing property of the $\Lambda$-spin is lost for these modes, and photon helicity extraction therefore requires known and finite $\Lambda_{b^{-}}$ polarization. For $\Lambda_{b}$ 's originating from energetic $b$-quarks heavy-quark effective theory predicts a large fraction of the longitudinal $b$-quark polarization to be retained after hadronization $[9,10]$. In fact, $\Lambda_{b}$-polarization is found to be substantial in $e^{+} e^{-} \rightarrow Z \rightarrow b \bar{b}$ reactions in agreement with the $Z b \bar{b}$-couplings of the SM [11]. There is no data yet on the polarization of $\Lambda_{b}$ 's produced in a hadronic environment. Expectations based on perturbative QCD combined with recent experimental data yield a polarization not exceeding the $10 \%$ level in high-energy $p p$ collisions, see Sec. 6. While the anomalously large polarization observed in $\Lambda$ production still lacks theoretical understanding, it could suggest larger polarization also for $\Lambda_{b}$ production.

The theoretical framework for radiative $\Lambda_{b}$ decays to $J=1 / 2 \Lambda$-baryons can be inferred from corresponding work on $\Lambda_{b} \rightarrow \Lambda(1116) \gamma$ decays, e.g., Ref. [5]. There is, however, no calculation available for $J=3 / 2$ (or higher). In particular, it is not known whether, for a given photon handedness, the decay amplitudes to the $\pm 1 / 2$ and $\pm 3 / 2$ helicity states differ significantly, as required for the extraction of the photon helicity along the lines of Ref. [7]. It is the purpose of this paper to fill this gap. Specifically, we will work out helicity amplitudes and rates for $\Lambda_{b} \rightarrow \Lambda(1520) \gamma$ decays. The $\Lambda(1520)$ with $J^{P}=3 / 2^{-}$is expected to produce a prominent peak in the $p K$-mass spec- 
trum from $\Lambda_{b} \rightarrow p K \gamma$ decays due to its large branching fraction to $p K$ and its relatively narrow width [7]. Soft-collinear effective theory (SCET) simplifies strong-interaction effects such as form factors in exclusive heavy-to-light decays at large recoil $[12,13]$. We work out lowest-order form factor relations in $\Lambda_{b} \rightarrow \Lambda(1520) \gamma$ decays and show that the amplitude with $\Lambda$-helicity $\pm 1 / 2$ dominates, thus supporting the experimental extraction of the photon helicity in $\Lambda_{b} \rightarrow \Lambda(J=3 / 2) \gamma \rightarrow p K \gamma$ modes according to Ref. [7]. Furthermore, we point out that measurements of $\Lambda_{b} \rightarrow \Lambda(1520) \gamma \rightarrow p K \gamma$ angular distributions afford a quantitative test of the suppression of the helicity-3/2 amplitude.

\section{$2 \quad \Lambda_{b} \rightarrow \Lambda(1520) \gamma$ amplitude and branching fraction}

In the following, we use $\Lambda$ to denote the $\Lambda$ (1520)-baryon unless otherwise stated. The description of weak decay amplitudes is done in an effective lowenergy theory framework [14] starting from the effective Hamiltonian

$$
\mathcal{H}_{\text {eff }}=-4 \frac{G_{F}}{\sqrt{2}} V_{t b} V_{t s}^{*} \sum c_{i} \mathcal{O}_{i}+c_{i}^{\prime} \mathcal{O}_{i}^{\prime}+\text { h.c. }
$$

where the chirality-flipped operators $\mathcal{O}_{i}^{\prime}$ are obtained from the $\mathrm{V}$-A-operators $\mathcal{O}_{i}$ by interchanging the chiral projectors $L / R=\left(1 \mp \gamma_{5}\right) / 2$. The most important contributions are due to the electromagnetic dipole operators

$$
\mathcal{O}_{7}=\frac{e}{16 \pi^{2}} m_{b} \bar{s} \sigma_{\mu \nu} F^{\mu \nu} R b, \quad \mathcal{O}_{7}^{\prime}=\frac{e}{16 \pi^{2}} m_{b} \bar{s} \sigma_{\mu \nu} F^{\mu \nu} L b,
$$

where $F^{\mu \nu}$ denotes the electromagnetic field strength tensor. Within the SM, the flipped dipole operator $\mathcal{O}_{7}^{\prime}$ is suppressed by the quark mass ratio $m_{s} / m_{b}$ with respect to $\mathcal{O}_{7}$, leading to predominantly left-handed photons in $b \rightarrow s \gamma$ quark decays.

The $\Lambda_{b} \rightarrow \Lambda(1520) \gamma$ amplitude can then be written as

$$
\begin{aligned}
i \mathcal{M}_{f i} & =\left\langle\gamma(q, \epsilon) \Lambda\left(p^{\prime}, s^{\prime}\right)\left|\mathcal{H}_{\mathrm{eff}}\right| \Lambda_{b}(p, s)\right\rangle \\
& =\kappa \cdot\left\{\left(C_{7}+C_{7}^{\prime}\right)\left\langle\Lambda\left|\bar{s} \sigma_{\mu \nu} q^{\mu} b\right| \Lambda_{b}\right\rangle+\left(C_{7}-C_{7}^{\prime}\right)\left\langle\Lambda\left|\bar{s} \sigma_{\mu \nu} q^{\mu} \gamma_{5} b\right| \Lambda_{b}\right\rangle\right\} \epsilon^{* \nu},
\end{aligned}
$$

where

$$
\kappa \equiv-i \frac{G_{F} V_{t b} V_{t s}^{*} e m_{b}}{\sqrt{2} 4 \pi^{2}}
$$

and $p(s), p^{\prime}\left(s^{\prime}\right)$ are the four-momenta (spins) of the $\Lambda_{b}, \Lambda$, respectively, and $q, \epsilon$ denote the photon momentum and polarization. In Eq. (3), $C_{7}, C_{7}^{\prime}$ (capital letters) are the effective coefficients of the corresponding dipole operators. At leading logarithmic order in $\alpha_{s}$, they coincide with the Wilson coefficients $c_{7}, c_{7}^{\prime}$ (small letters), respectively, up to small contributions from penguin operators, and are understood to be evaluated at the $m_{b}$-scale. 
We write the hadronic matrix elements in terms of the tensors $\Gamma$ and $\Gamma_{(5)}$ defined as

$$
\begin{aligned}
\left\langle\Lambda\left|\bar{s} \sigma_{\mu \nu} q^{\mu} b\right| \Lambda_{b}\right\rangle & \equiv \bar{\Psi}^{\alpha}\left(p^{\prime}, s^{\prime}\right) \Gamma_{\alpha \nu} u\left(p^{\prime}+q, s\right), \\
\left\langle\Lambda\left|\bar{s} \sigma_{\mu \nu} q^{\mu} \gamma_{5} b\right| \Lambda_{b}\right\rangle & \equiv \bar{\Psi}^{\alpha}\left(p^{\prime}, s^{\prime}\right) \Gamma_{(5) \alpha \nu} u\left(p^{\prime}+q, s\right),
\end{aligned}
$$

where $\Psi^{\alpha}$ denotes the Rarita-Schwinger spinor of the $\Lambda(1520)$ and $u$ the $\Lambda_{b^{-}}$ spinor. To describe decays into on-shell photons, we use the following covariants with two form factors $f_{i} \equiv f_{i}\left(q^{2}=0\right), i=1,2$ :

$$
\begin{aligned}
\Gamma_{\alpha \nu} & =\frac{f_{1}}{m_{\Lambda_{b}}}\left(q_{\alpha} p_{\nu}^{\prime}-g_{\alpha \nu} p^{\prime} \cdot q\right)+f_{2}\left(q_{\alpha} \gamma_{\nu}-g_{\alpha \nu} \not q\right), \\
\Gamma_{(5) \alpha \nu} & =\frac{f_{1}}{m_{\Lambda_{b}}}\left(q_{\alpha} p_{\nu}^{\prime}-g_{\alpha \nu} p^{\prime} \cdot q\right) \gamma_{5}-\left(\frac{m_{\Lambda}}{m_{\Lambda_{b}}} f_{1}+f_{2}\right)\left(q_{\alpha} \gamma_{\nu}-g_{\alpha \nu} \not q\right) \gamma_{5} .
\end{aligned}
$$

Here, $m_{\Lambda_{b}}, m_{\Lambda}$ denote the masses of the $\Lambda_{b^{-}}$and $\Lambda$-baryon, respectively. The vertices $\Gamma_{\alpha \nu}$ and $\Gamma_{(5) \alpha \nu}$ are related due to the identity $\sigma_{\mu \nu} \gamma_{5}=-\frac{i}{2} \epsilon_{\mu \nu \alpha \beta} \sigma^{\alpha \beta}$. In writing down Eqs. (7) and (8), we used the equations of motion, gauge invariance $\Gamma_{\alpha \nu} q^{\nu}=0$ and $\Gamma_{(5) \alpha \nu} q^{\nu}=0$, parity conservation of the strong interaction-for a $(1 / 2)^{+} \rightarrow(3 / 2)^{-}$transition $\Gamma_{\alpha \nu}$ is P-even and $\Gamma_{(5) \alpha \nu} \mathrm{P}$ odd - and the conditions

$$
\gamma_{\alpha} \Psi^{\alpha}=0, \quad p_{\alpha}^{\prime} \Psi^{\alpha}=0
$$

For other Lorentz decompositions of $\Gamma, \Gamma_{(5)}$ we refer to Ref. [15].

We proceed to calculate the spin-averaged branching fraction for $\Lambda_{b} \rightarrow \Lambda(1520) \gamma$ decays. Employing the Rarita-Schwinger spin summation formula:

$$
\sum_{s^{\prime}} \Psi_{\alpha}\left(p^{\prime}, s^{\prime}\right) \bar{\Psi}_{\beta}\left(p^{\prime}, s^{\prime}\right)=-\left(\not p^{\prime}+m_{\Lambda}\right)\left\{g_{\alpha \beta}-\frac{\gamma_{\alpha} \gamma_{\beta}}{3}-\frac{2 p_{\alpha}^{\prime} p_{\beta}^{\prime}}{3 m_{\Lambda}^{2}}+\frac{p_{\alpha}^{\prime} \gamma_{\beta}-p_{\beta}^{\prime} \gamma_{\alpha}}{3 m_{\Lambda}}\right\},
$$

we obtain for the total spin-averaged branching fraction with the $\Lambda_{b}$-lifetime $\tau_{\Lambda_{b}}$

$$
\mathcal{B}\left(\Lambda_{b} \rightarrow \Lambda(1520) \gamma\right)=\tau_{\Lambda_{b}}\left(1-\frac{m_{\Lambda}^{2}}{m_{\Lambda_{b}}^{2}}\right) \frac{\left|\mathcal{M}_{\mathrm{ave}}\right|^{2}}{16 \pi m_{\Lambda_{b}}}
$$

where

$$
\begin{aligned}
\left|\mathcal{M}_{\mathrm{ave}}\right|^{2}= & \frac{1}{2} \sum_{\text {spins }}\left|\mathcal{M}_{f i}\right|^{2} \\
= & \frac{2}{3} \frac{m_{\Lambda_{b}}^{6}}{m_{\Lambda}^{2}}\left(1-\frac{m_{\Lambda}^{2}}{m_{\Lambda_{b}}^{2}}\right)^{2}|\kappa|^{2}\left(\left|C_{7}\right|^{2}+\left|C_{7}^{\prime}\right|^{2}\right)\left[f_{1}^{2}\left(\frac{m_{\Lambda}}{m_{\Lambda_{b}}}\right)^{2}\left(1+\frac{m_{\Lambda}}{m_{\Lambda_{b}}}\right)^{2}\right. \\
& \left.+f_{1} f_{2} \frac{m_{\Lambda}}{m_{\Lambda_{b}}}\left(1+4 \frac{m_{\Lambda}}{m_{\Lambda_{b}}}+3 \frac{m_{\Lambda}^{2}}{m_{\Lambda_{b}}^{2}}\right)+f_{2}^{2}\left(1+3 \frac{m_{\Lambda}^{2}}{m_{\Lambda_{b}}^{2}}\right)\right]
\end{aligned}
$$


In the limit $m_{\Lambda} \ll m_{\Lambda_{b}}$ this yields

$$
\mathcal{B}\left(\Lambda_{b} \rightarrow \Lambda(1520) \gamma\right)=\tau_{\Lambda_{b}}\left(1-\frac{m_{\Lambda}^{2}}{m_{\Lambda_{b}}^{2}}\right)^{3} \frac{m_{\Lambda_{b}}^{5}}{m_{\Lambda}^{2}} \frac{\alpha G_{F}^{2}\left|V_{t b} V_{t s}^{*}\right|^{2} m_{b}^{2}}{192 \pi^{4}} f_{2}^{2}\left(\left|C_{7}\right|^{2}+\left|C_{7}^{\prime}\right|^{2}\right)
$$

where we kept the full phase space factor.

There is no information on the form factors $f_{1,2}$ currently available. In Sec. 4 we will work out a relation between them. Since $\left|C_{7}\right|^{2}+\left|C_{7}^{\prime}\right|^{2}$ is strongly constrained to be close to its SM value by data on $B \rightarrow X_{s} \gamma$ decays, a future measurement of the $\Lambda_{b} \rightarrow \Lambda(1520) \gamma$ branching fraction will determine $f_{2}$, see Eq. (13).

Beyond lowest order, the relation between the genuine short-distance coefficients from $\mathcal{H}_{\text {eff }}, c_{7}^{(\prime)}$, and the effective coefficients, $C_{7}^{(\prime)}$, is modified by calculable perturbative $\mathcal{O}\left(\alpha_{s}\right)$-corrections to the vertex, hard scattering and annihilation contributions, see, e.g., Ref. [16] for the corresponding analysis of $B \rightarrow K^{*} \gamma$ decays, and also Ref. [17] for contributions beyond QCD factorization. Fully-fledged calculations for $b$-baryon decays are not available, but we can still make some remarks: The vertex correction at next-to-leading order has already been estimated in Ref. [5] for $\Lambda_{b} \rightarrow \Lambda(1116) \gamma$ decays. We expect a similar correction for $\Lambda_{b} \rightarrow \Lambda(1520) \gamma$. As for the contributions beyond the soft form factor, the hard-scattering scale for heavy-to-light baryons at large recoil is lowered with respect to the one for mesons due to the larger number of constituents, hence will induce parametrically larger $\alpha_{s}$-corrections. Weak annihilation contributions to $b \rightarrow s$-transitions are CKM-suppressed by $V_{u b} V_{u s}^{*} / V_{t b} V_{t s}^{*}$, but arise at tree level from $W$-boson exchange. The situation regarding weak annihilation improves for baryons, where such contributions are colour-suppressed.

At higher order, the effective coefficients develop also an absorptive part, and allow for CP violation in decay, see Ref. [5] for a discussion in $\Lambda_{b} \rightarrow \Lambda(1116) \gamma$ decays. Similarly to final state $\Lambda(1116)$ 's, the heavier $\Lambda$ resonances decaying to $p K$ are self-tagging, i.e., $\Lambda$ decays to $K^{-}$whereas $\bar{\Lambda}$ decays to $K^{+}$.

\section{Helicity amplitudes for $\Lambda_{b} \rightarrow \Lambda(1520) \gamma$}

We decompose the decay amplitude into helicity amplitudes $\mathcal{A}_{h}$, labelled by the $\Lambda$-helicity $h$, where $h= \pm 1 / 2, \pm 3 / 2$ :

$$
\sum_{\text {spins }}\left|\mathcal{M}_{f i}\right|^{2}=\sum_{h= \pm 1 / 2, \pm 3 / 2}\left|\mathcal{A}_{h}\right|^{2} .
$$


We calculate the amplitudes $\mathcal{A}_{h}$ in the $\Lambda_{b}$-rest frame, where we choose the momentum of the $\Lambda$ to be in the $+z$-direction, and use

$$
\begin{array}{rlrl}
p^{\prime \mu} & =\left(E^{\prime}, 0,0, E\right), & q^{\mu}=(E, 0,0,-E), \\
E & =\frac{m_{\Lambda_{b}}^{2}-m_{\Lambda}^{2}}{2 m_{\Lambda_{b}}}, & E^{\prime} & =\frac{m_{\Lambda_{b}}^{2}+m_{\Lambda}^{2}}{2 m_{\Lambda_{b}}},
\end{array}
$$

as well as $\epsilon_{ \pm 1}^{* \mu}=\mp 1 / \sqrt{2}(0,1, \mp i, 0)$ for a photon with angular momentum in the $+z$ direction, i.e., $J_{z}= \pm 1$ and $q \cdot \epsilon^{*}=0$. Note that in our reference frame we also have $p^{\prime} \cdot \epsilon_{ \pm}^{*}=0$. The $\Lambda$-polarization vectors $\omega_{i}$ with helicity $i= \pm 1,0$ are given as

$$
\omega_{ \pm 1}^{* \mu}=\mp \frac{1}{\sqrt{2}}(0,1, \mp i, 0), \quad \omega_{0}^{* \mu}=\frac{1}{m_{\Lambda}}\left(E, 0,0, E^{\prime}\right)
$$

with $p^{\prime} \cdot \omega_{i}^{*}=0$. Then we write the Rarita-Schwinger spinor $\Psi_{h}^{\alpha}$ of the $\Lambda(1520)$ with helicity $h= \pm 1 / 2, \pm 3 / 2$ as

$$
\begin{aligned}
& \Psi_{ \pm 3 / 2}^{\alpha}=\Psi_{ \pm 1 / 2} \cdot \omega_{ \pm 1}^{\alpha}, \\
& \Psi_{ \pm 1 / 2}^{\alpha}=\sqrt{\frac{2}{3}} \Psi_{ \pm 1 / 2} \cdot \omega_{0}^{\alpha}+\sqrt{\frac{1}{3}} \Psi_{\mp 1 / 2} \cdot \omega_{ \pm 1}^{\alpha},
\end{aligned}
$$

where in abuse of notation we denote by $\Psi_{h}$ the spin- $1 / 2$ component of the $\Lambda$ with helicity $h= \pm 1 / 2$.

The amplitudes $\mathcal{A}_{ \pm 3 / 2}\left(\mathcal{A}_{ \pm 1 / 2}\right)$ result from a $\Lambda_{b}$-baryon with $h= \pm 1 / 2(h=$ $\mp 1 / 2$ ) and a photon with $J_{z}=\mp 1$, i.e., right- or left-handed, respectively. We arrive at the following helicity amplitudes for $\Lambda_{b} \rightarrow \Lambda(1520) \gamma$ decays:

$$
\begin{aligned}
& \mathcal{A}_{+3 / 2}=-2 \kappa\left(m_{\Lambda_{b}}^{2}-m_{\Lambda}^{2}\right)\left[\frac{m_{\Lambda_{b}}+m_{\Lambda}}{2 m_{\Lambda_{b}}} f_{1}+f_{2}\right] C_{7}^{\prime}, \\
& \mathcal{A}_{+1 / 2}=-\frac{2 \kappa}{\sqrt{3}}\left(m_{\Lambda_{b}}^{2}-m_{\Lambda}^{2}\right)\left[\frac{m_{\Lambda_{b}}+m_{\Lambda}}{2 m_{\Lambda_{b}}} f_{1}+\frac{m_{\Lambda_{b}}}{m_{\Lambda}} f_{2}\right] C_{7}^{\prime}, \\
& \mathcal{A}_{-1 / 2}=-\frac{2 \kappa}{\sqrt{3}}\left(m_{\Lambda_{b}}^{2}-m_{\Lambda}^{2}\right)\left[\frac{m_{\Lambda_{b}}+m_{\Lambda}}{2 m_{\Lambda_{b}}} f_{1}+\frac{m_{\Lambda_{b}}}{m_{\Lambda}} f_{2}\right] C_{7}, \\
& \mathcal{A}_{-3 / 2}=-2 \kappa\left(m_{\Lambda_{b}}^{2}-m_{\Lambda}^{2}\right)\left[\frac{m_{\Lambda_{b}}+m_{\Lambda}}{2 m_{\Lambda_{b}}} f_{1}+f_{2}\right] C_{7} .
\end{aligned}
$$

Evaluating Eq. (14), we recover the result for the squared spin-averaged matrix element given in Eq. (12). The $1 / m_{\Lambda}$ factor in $\mathcal{A}_{ \pm 1 / 2}$ results from the longitudinal polarization vector of the $\Lambda$-baryon, $\omega_{0}$. It implies a (kinematical) suppression of the helicity-3/2 amplitude with respect to the helicity- $1 / 2$ one by

$$
\frac{\mathcal{A}_{ \pm 3 / 2}}{\mathcal{A}_{ \pm 1 / 2}} \simeq \sqrt{3} \frac{m_{\Lambda}}{m_{\Lambda_{b}}}\left(1+\frac{f_{1}}{2 f_{2}}\right)
$$

up to corrections of higher order in $m_{\Lambda} / m_{\Lambda_{b}}$. 


\section{$4 \Lambda$ helicity at large recoil and experimental test}

Generally, we expect the 3/2-amplitude to be power-suppressed due the enforced change of helicity of the light degrees of freedom in the decay $[18,19]$. This can be shown explicitly using symmetry relations for heavy-to-light currents arising when the emitted light hadron is energetic [12]. In the symmetry limit, we obtain for $\Lambda_{b} \rightarrow \Lambda(1520)$ processes with arbitrary Dirac structure $\Gamma$ :

$$
\begin{aligned}
\left\langle\Lambda\left|\bar{\chi} \Gamma b_{v}\right| \Lambda_{b}\right\rangle & =\bar{\Psi}^{\alpha}\left(n, s^{\prime}\right) q_{\alpha} X \Gamma u(v, s) \\
& =\bar{\Psi}^{\alpha}\left(n, s^{\prime}\right) q_{\alpha}(A+B \not \nu) \Gamma u(v, s),
\end{aligned}
$$

where $b_{v}$ is a heavy quark with velocity $v$ and $\chi$ a strange-flavoured collinear field associated with the light-like momentum $n=p^{\prime} / E^{\prime}, n^{2} \simeq 0$ living in the effective theory (SCET) [13]. Wilson lines are understood in the definition of $\chi$. In the second step, we have expressed the bispinor $X$ through a most general, independent ansatz out of $v, n$ in terms of two form factors $A, B$, both functions of $E^{\prime}$. For radiative decays to on-shell photons with dipole currents $\Gamma=\sigma_{\mu \nu} q^{\mu}\left(\gamma_{5}\right)$ this implies only one single Dirac structure

$$
\Gamma_{\alpha \nu}=\left(A+B \frac{m_{\Lambda}}{m_{\Lambda_{b}}}\right) q_{\alpha} \sigma_{\mu \nu} q^{\mu}
$$

and an analogous equation for $\Gamma_{(5)}$. Comparing this to the full QCD formulae, Eqs. (7) and (8), we obtain a relation between the form factors $f_{1}$ and $f_{2}$ :

$$
f_{1}=-f_{2} \frac{2 m_{\Lambda_{b}}}{m_{\Lambda_{b}}+m_{\Lambda}} .
$$

Hence, at lowest order in SCET, the 3/2-amplitudes vanish, see Eqs. (20) and (23). Similar to the analogous relations based on the helicity conservation property of the strong interaction in $B$ decays to vectors [19,20], Eq. (27) should hold to all orders in $\alpha_{s}$. We expect that finite $\mathcal{A}_{ \pm 3 / 2}$ arises from $1 / E^{\prime}$ corrections to Eq. (27).

The form factor relation given in Eq. (27) is a central result of our work. It enables the extraction of the ratio $\left|C_{7}^{\prime} / C_{7}\right|$ from $\Lambda_{b}$ decays to spin-3/2 $\Lambda$ baryons as advocated in Ref. [7], which requires disparate helicity amplitudes. Indeed, the squared amplitude ratio $\eta$, defined as

$$
\eta \equiv \frac{\left|\mathcal{A}_{ \pm 3 / 2}\right|^{2}}{\left|\mathcal{A}_{ \pm 1 / 2}\right|^{2}}
$$

and introduced in Ref. [7] to parameterize the suppression in sensitivity to $\left|C_{7}^{\prime} / C_{7}\right|$ from a possible dilution due to finite helicity-3/2 amplitude, turns out to be zero at lowest order SCET:

$$
\eta=0
$$


We expect finite $\eta$ at the order $\left(m_{\Lambda} / m_{\Lambda_{b}} \times \Lambda_{\mathrm{QCD}} / E^{\prime}\right)^{2}$ not exceeding the percent-level.

Interestingly the parameter $\eta$ can be determined experimentally from

$$
\frac{d \Gamma}{d \cos \theta_{p}} \propto 1-\alpha_{p, 3 / 2} \cos ^{2} \theta_{p}, \quad \text { with } \quad \alpha_{p, 3 / 2}=\frac{\eta-1}{\eta+\frac{1}{3}},
$$

where $\theta_{p}$ denotes the angle between the $\Lambda$ direction and the proton momentum in the $\Lambda$-rest frame [7]. We find that a sample of $10^{4} \Lambda_{b} \rightarrow \Lambda(1520) \gamma \rightarrow p K \gamma$ decays, equivalent to about three years of data-taking with the LHCb detector [21], would yield a statistical precision of 0.03 on $\eta$. With a clear-cut prediction for $\eta$ at hand, this measurement would provide a valuable benchmark for SCET.

\section{Photon helicity analysis}

With known and finite $\Lambda_{b}$ polarization $P_{\Lambda_{b}}$ the helicity of the emitted photon, and hence the ratio $\left|C_{7}^{\prime} / C_{7}\right|$, is extracted from the differential $\Lambda_{b} \rightarrow \Lambda(1520) \gamma$ rate (see Ref. [7] for details),

$$
\frac{d \Gamma}{d \cos \theta_{\gamma}} \propto 1-\alpha_{\gamma, 3 / 2} P_{\Lambda_{b}} \cos \theta_{\gamma}
$$

where the angle $\theta_{\gamma}$ between the $\Lambda_{b}$-spin and the direction of the photon momentum is defined in the $\Lambda_{b}$-rest frame. For example, the SM-type amplitudes $\mathcal{A}_{-1 / 2}\left(\mathcal{A}_{-3 / 2}\right)$ predominantly yield $\theta_{\gamma} \approx \pi(0)$. The photon asymmetry parameter $\alpha_{\gamma, 3 / 2}$ provides the link between experiment and theory. It is given as

$$
\alpha_{\gamma, 3 / 2}=\frac{1-\eta}{1+\eta} \cdot \frac{\left|C_{7}\right|^{2}-\left|C_{7}^{\prime}\right|^{2}}{\left|C_{7}\right|^{2}+\left|C_{7}^{\prime}\right|^{2}}
$$

with $\eta$ defined in Eq. (28). It is clear from Eq. (32) that the sensitivity to $\left|C_{7}^{\prime} / C_{7}\right|$ would vanish for $\eta \approx 1$. Now, according to Sec. $4 \eta \approx 0$ and the extraction of $\left|C_{7}^{\prime} / C_{7}\right|$ from a measurement of $d \Gamma / d \cos \theta_{\gamma}$ is feasible without restriction. In Fig. 1 we show the expected experimental sensitivity to righthanded currents in the case $\eta \approx 0\left(f_{1} / f_{2} \approx-2\right)$ as a function of the $\Lambda_{b}$ polarization $P_{\Lambda_{b}}$ for decays to $\Lambda(1520)$ and $\Lambda(1690)$. The curves show the minimum $\left|C_{7}^{\prime} / C_{7}\right|$ accessible at $3 \sigma$ (standard deviation) significance under the statistics assumptions of Ref. [7], i.e., for a generic hadron collider experiment capable of collecting $10^{4} \Lambda_{b} \rightarrow \Lambda(1520) \gamma \rightarrow p K \gamma$ decays. The event yields underlying our sensitivity estimates are therefore simply $10^{4}$ for $\Lambda(1520)$ and $10^{4}$ scaled by the expected branching ratios $[5,7]$ for other $\Lambda$ 's. The significance 


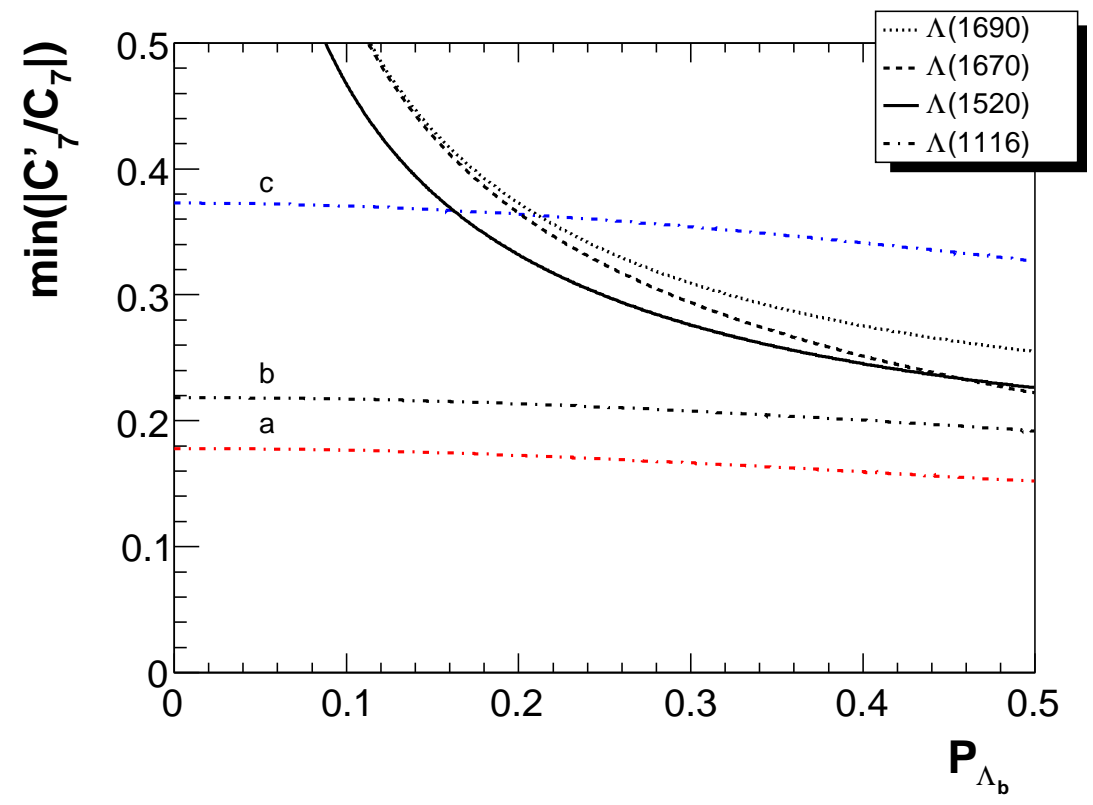

Fig. 1. Experimental reach for the ratio $\left|C_{7}^{\prime} / C_{7}\right|$ as a function of the $\Lambda_{b}$ polarization $P_{\Lambda_{b}}$ for the decays $\Lambda_{b} \rightarrow \Lambda(X) \gamma \rightarrow p K \gamma, X=1520,1670,1690$. For comparison, the corresponding reach of the $\Lambda_{b} \rightarrow \Lambda(1116) \gamma \rightarrow p \pi \gamma$ decay is shown for the reconstruction scenarios (a)-(c) described in the text. The curves indicate the minimum $\left|C_{7}^{\prime} / C_{7}\right|$ accessible at $3 \sigma$ (standard deviation) significance at a hadron collider experiment after collecting statistics equivalent to $10^{4} \Lambda_{b} \rightarrow \Lambda(1520) \gamma \rightarrow p K \gamma$ decays.

is determined as $\left(1-\alpha_{\gamma, \frac{3}{2}}\right) / \sigma_{\alpha_{\gamma, \frac{3}{2}}}$ (or equivalent for decays to spin- $1 / 2$ baryons), see Erratum to Ref. [7].

For comparison, the curves for decays to the spin- $1 / 2$ resonance $\Lambda(1670)$ and to the ground state $\Lambda(1116)$, with $\Lambda(1116) \rightarrow p \pi$, are also shown. In the latter case, we illustrate the effect of event losses from trigger and reconstruction difficulties by considering three scenarios in which the reconstruction efficiency for $\Lambda_{b} \rightarrow \Lambda(1116) \gamma \rightarrow p \pi \gamma$ is (a) equal, (b) worse by factor of 10, and (c) worse by a factor of 100 with respect to $\Lambda_{b} \rightarrow \Lambda(X) \gamma \rightarrow p K \gamma$. The expected reduction of the reconstruction efficiency is primarily caused by the lack of an observable decay vertex in the innermost part of the detector.

Thanks to the suppression of the helicity-3/2 amplitude, radiative $\Lambda_{b}$ decays to spin-3/2 resonances suffer almost no loss in sensitivity to $\left|C_{7}^{\prime} / C_{7}\right|$ with respect to decays to spin- $1 / 2$ resonances for $\Lambda_{b}$ polarization not exceeding about 0.2 . This is of vital importance since the cleanest and statistically most fertile among the $\Lambda_{b} \rightarrow p K \gamma$ resonance decays proceeds via the $\Lambda(1520)$, which 
has spin $3 / 2$. For larger $\Lambda_{b}$ polarization, the statistical error on the proton asymmetry parameter curbs the reach of decays to spin-3/2 resonances in comparison to decays to spin- $1 / 2$ resonances. For instance for $P_{\Lambda_{b}} \approx 0.46$ and higher, the decay to $\Lambda(1670)$ can probe for lower $\left|C_{7}^{\prime} / C_{7}\right|$ than the decay to $\Lambda(1520)$, despite the inferior event yield of the $\Lambda(1670)$, see Fig. 1.

\section{$6 \quad \Lambda_{b}$-polarization in hadronic collisions}

The extraction of the photon helicity from radiative $\Lambda_{b}$-decays to $\Lambda$ 's heavier than the $1116-\mathrm{MeV}$ ground state hinges crucially on the value of $\Lambda_{b}$ polarization $P_{\Lambda_{b}}$. We seize this opportunity to briefly revisit the mechanism responsible for heavy baryon polarization at hadron colliders.

$\Lambda_{b}$-polarization is a consequence of finite $b$-quark polarization. The latter arises in (unpolarized) $p \bar{p}-, p p$-collisions from QCD through its well-known mechanism of inducing CP-odd observables such as transversal spin asymmetries $\vec{s} \cdot\left(\vec{n}_{1} \times \vec{n}_{2}\right)$ from strong phases. We denote by $\sigma(\uparrow)(\sigma(\downarrow))$ the single quark production cross sections with the quark spin $\vec{s}$ being up (down) with respect to the scattering plane $\vec{n}_{1} \times \vec{n}_{2}$. Here, for example, the unit vectors $\vec{n}_{1}$ and $\vec{n}_{2}$ could be chosen along the beam direction and in the direction of the produced quark or hadron, respectively. This leads to the transversal quark-polarization

$$
P_{q} \equiv \frac{\sigma(\uparrow)-\sigma(\downarrow)}{\sigma(\uparrow)+\sigma(\downarrow)} .
$$

In high energy reactions, $P_{q}$ can be calculated in QCD-perturbation theory [22]. Since it involves a quark helicity change, it is proportional to the quark mass. $P_{q}$ depends non-trivially on the kinematics, such as the centre-of-mass energy and scattering angle $\theta$. Note that for $\theta \rightarrow 0$ or $\pi, P_{q}$ vanishes due to the absence of a scattering plane and emergent rotational invariance. A finite $P_{q}$ arises at one-loop order in the strong interaction, hence $P_{q} \propto \alpha_{s} m_{q}$. From explicit calculation the largest polarization for $b$-quarks is found to be $\mathcal{O}(10 \%)$ [22]. The dependence of $P_{b}$ on the kinematics could also result in different values of $P_{\Lambda_{b}}$ if measured in different kinematical regions or experimental setups. This deserves further study.

Finite polarization of $\Lambda_{b}$-baryons is then inherited from the $b$-quarks as a fraction of the $b$-quark polarization. To what extent depends on whether the $\Lambda_{b}$ 's hadronize directly from the $b$-quarks $\left(P_{\Lambda_{b}}=P_{b}\right.$ in the heavy quark limit [9]),

or are produced via $\Sigma_{b}^{(*)}$-baryons, which decay strongly to $\Lambda_{b} \pi[10]$. In the latter case, the amount of depolarization can be expressed in terms of two fragmentation parameters in the limit of completely incoherently decaying $\Sigma_{b}$, $\Sigma_{b}^{*}$-resonances. There is a lower bound $P_{\Lambda_{b}} / P_{b} \geq 1 / 9$, but more common val- 
ues for the parameters yield much larger $P_{\Lambda_{b}} / P_{b}$, e.g., $72 \%$ [10], which is also supported by LEP data [11]. Besides fragmentation, the amount of depolarization also depends on how fast the $\left(\Sigma_{b}, \Sigma_{b}^{*}\right)$-multiplet decays with respect to its energy splitting $\Delta$ : first, the longer the $\Sigma_{b}^{(*)}$-resonances live, the more time there is for the heavy quark spin to interact with the light degrees of freedom and, second, the further apart the resonances are within the multiplet, the larger the heavy quark spin interaction. Hence the $\Lambda_{b}$-polarization increases with $\Gamma\left(\Sigma_{b}^{(*)} \rightarrow \Lambda_{b} \pi\right) / \Delta$. The dependence of the polarization on $\Gamma / \Delta$ has been modelled by Falk and Peskin [10].

Recently, the CDF collaboration reported a first measurement of the mass splitting of the $\Sigma_{b}^{(*)}$-doublet [23]

$$
\Delta \equiv m_{\Sigma_{b}^{* \mp}}-m_{\Sigma_{b}^{\mp}}=21.3_{-1.9-0.2}^{+2.0+0.4} \mathrm{MeV} .
$$

In absence of a measurement the rate for $\Sigma_{b}^{(*)} \rightarrow \Lambda_{b} \pi$ decays can be calculated using heavy quark symmetries [24]

$$
\Gamma \equiv \Gamma\left(\Sigma_{b}^{(*)} \rightarrow \Lambda_{b} \pi\right)=\frac{1}{6 \pi} \frac{m_{\Lambda_{b}}}{m_{\Sigma_{b}^{(*)}}} \frac{g_{A}^{2}}{f_{\pi}^{2}}\left|\vec{p}_{\pi}\right|^{3},
$$

where $g_{A}=0.75$ is a phenomenological coupling of the constituent quark, $f_{\pi}=$ $92.4 \mathrm{MeV}[8]$ is the pion decay constant, and $\vec{p}_{\pi}$ denotes the pion momentum in the $\Sigma_{b}$ centre-of-mass frame. Note that the decay rates are equal up to higher order $1 / m_{b}$ corrections. Numerically the range $5808-5837 \mathrm{MeV}$ for $\Sigma_{b}^{(*)}$ masses [23] yields $6.5 \mathrm{MeV}<\Gamma<14.7 \mathrm{MeV}$, therefore $0.3<\Gamma / \Delta<0.7$. With rate and splitting being of the same order, the $\Sigma_{b}, \Sigma_{b}^{*}$-resonances are partly overlapping, and the depolarization of the final $\Lambda_{b}$ 's is reduced with respect to the $\Gamma / \Delta \ll 1$ limit.

With this new information, we update the result from Ref. [10], using the same values for the fragmentation parameters, and find

$$
74 \%<\frac{P_{\Lambda_{b}}}{P_{b}}<81 \%
$$

This range should be seen as a first order estimate; it has uncertainties from the fragmentation parameters and the extrapolation to realistic values for $\Gamma$ and $\Delta$.

We are aware that perturbative QCD is not sufficient to explain the observed huge strange hyperon polarization [25]. Given the lack of a model-independent description, however, which would then also apply to heavy baryons, we do not draw any conclusions for the polarization of the $\Lambda_{b}$ 's.

Experimentally, $P_{\Lambda_{b}}$ is expected to be measured with a precision of $\sigma\left(P_{\Lambda_{b}}\right) \approx$ 0.016 at the LHC [26]. 


\section{Summary and Outlook}

Motivated by its prospective use as a probe for right-handed currents in $b \rightarrow s \gamma$ we have worked out parameterizations for branching fraction and helicity amplitudes of the decay $\Lambda_{b} \rightarrow \Lambda(1520) \gamma$. Our framework applies to radiative $\Lambda_{b^{-}}$ decays to any strange isosinglet baryon with $J^{P}=3 / 2^{-}$, that is, the $\Lambda(1520)$, $\Lambda(1690)$ and so on. Formulae for the even-parity $\Lambda$-baryons with $J^{P}=3 / 2^{+}$ can be obtained by interchanging the right-hand side of Eq. (7) with the one of Eq. (8). In the final formulae, Eqs. (11-13) and Eqs. (20-23), this corresponds to replacing $C_{7}^{\prime}$ by $-C_{7}^{\prime}$.

In the approximation of energetic light-hadron emission (SCET) we find the helicity-3/2 amplitude to vanish at lowest order, a result which simplifies considerably the experimental extraction of the photon helicity in that mode, as shown in Fig. 1. The predicted suppression of the helicity-3/2 amplitude is readily testable at collider experiments and therefore could serve as a reference point for SCET and its applicability to baryons. We have also re-estimated $\Lambda_{b}$-polarization at hadron colliders, updating the prediction from heavy-quark effective theory with recent data on $\Sigma_{b}^{(*)}$ resonances from the CDF collaboration.

Given sufficient data, the experimental investigations of radiative $\Lambda_{b}$ decays can contribute further to the general programme of flavour physics [1]: There is the possibility of searching for CP violation beyond the SM by measuring the branching fractions and photon polarization asymmetries $\alpha_{\gamma, 3 / 2}$ in $\Lambda_{b} \rightarrow$ $\Lambda(1520) \gamma$ and its CP conjugated mode $\bar{\Lambda}_{b} \rightarrow \bar{\Lambda}(1520) \gamma$ separately. In the absence of new CP phases, the respective observables from $b$ and $\bar{b}$ decay are equal up to a very small SM background of the order $\operatorname{Im}\left(V_{u s}^{*} V_{u b} / V_{t s}^{*} V_{t b}\right)$. Note that a non-zero $\mathrm{CP}$ asymmetry in both cases requires a finite strong phase, which arises at higher order, see Sec. 2. For the $\Lambda_{b}-\bar{\Lambda}_{b}$ rate asymmetry, new physics signals are already strongly constrained by $B \rightarrow K^{*} \gamma$ decays [3], but in the presence of right-handed currents, the asymmetry $\alpha_{\gamma, 3 / 2}$ in baryon decays would provide complementary information. This has been worked out and discussed in detail for the case of the $\Lambda(1116)$ in Ref. [5] and can be applied to heavier $\Lambda$ 's accordingly.

Another application for $\Lambda_{b}$ samples of very high statistics are investigations of the $b \rightarrow d \gamma$ penguin with $\Lambda_{b} \rightarrow N(X) \gamma \rightarrow p \pi \gamma$ decays. Indeed, a primary goal of flavour physics is to find out whether the Yukawa couplings are the only source of flavour and CP violation or whether there are new such sources [27]. The former case, which includes the SM, is termed Minimal Flavour Violation [28] and subject to intense ongoing tests. In this scenario, all flavour-changing processes are related via the CKM-link, in particular the $b \rightarrow s \gamma$ and $b \rightarrow d \gamma$ transitions. We therefore also suggest the study of $\Lambda_{b} \rightarrow N(X) \gamma \rightarrow p \pi \gamma$ 
decays in analogy to $\Lambda_{b} \rightarrow \Lambda(X) \gamma \rightarrow p K \gamma$, that is, to measure branching fractions and photon polarization. The $\Lambda_{b} \rightarrow N(X) \gamma$ modes offer one of the rare opportunities to obtain information on right-handed currents in the $b \rightarrow$ $d \gamma$ transition, another one being time-dependent CP asymmetries in $B$-meson decays to $\mathrm{CP}$ eigenstates [29]. We expect to see the $b \rightarrow d \gamma$ transition in radiative $\Lambda_{b}$ decays with branching fractions of a few $10^{-7}-10^{-6}$, lowered by $\left|V_{t d} / V_{t s}\right|^{2} \approx 1 / 25$ with respect to the corresponding $b \rightarrow s \gamma$ modes and in agreement with $B \rightarrow\left(K^{*}, \rho\right) \gamma$ decays [3]. Currently, the $b \rightarrow d \gamma$ penguin is only poorly known, and any bound on its chirality would add information. The observation of a discrepancy between $C_{7}^{\prime} / C_{7}$ extracted from $b \rightarrow s$ versus $b \rightarrow d$ modes 2 would be a clean signal of breakdown of the SM and more generally Minimal Flavour Violation, where at the perturbative level

$$
\left|\frac{C_{7}^{\prime}}{C_{7}}\right|_{b \rightarrow s}-\left|\frac{C_{7}^{\prime}}{C_{7}}\right|_{b \rightarrow d}=\mathcal{O}\left(\frac{m_{s}-m_{d}}{m_{b}}\right) \simeq \mathcal{O}\left(\frac{m_{s}}{m_{b}}\right) .
$$

Allowing also for non-perturbative effects, the difference is still protected by U-spin symmetry, which is only mildly broken, for example by the difference between baryon masses. In addition, Eq. (37) will receive corrections $\propto\left(V_{u b} V_{u d}^{*} / V_{t b} V_{t d}^{*}\right)\left(\Lambda_{\mathrm{QCD}} / m_{b}\right)$ from annihilation and up-charm loops with treelevel induced Wilson coefficient, which are not CKM suppressed in the $b \rightarrow d$ transition [30]. Studies in $B \rightarrow\left(K^{*}, \rho\right) \gamma$ decays show that these corrections do not spoil the suppression of right-handed photons in $b \rightarrow(s, d) \gamma$ in the SM and give $\left|C_{7}^{\prime} / C_{7}\right|_{b \rightarrow(s, d)}$ of comparable order of magnitude $[30,17]$.

We would like to stress here that due to the interference between mixing and decay, time-dependent CP asymmetries in meson decays can only probe contributions with weak CP phase other than the one of the meson mixing amplitude. On the other hand, the photon polarization analysis in $\Lambda_{b} \rightarrow$ $\Lambda(X) \gamma, N(X) \gamma$ is sensitive to the magnitudes of the total amplitudes $\left|C_{7}^{(\prime)}\right|$. We conclude that radiative $\Lambda_{b}$ decays constitute a rich testing ground for physics within and beyond the Standard Model.

\section{Acknowledgements}

The work of G.H. is supported in part by Bundesministerium für Bildung und Forschung, Berlin/Bonn. T.S. gratefully acknowledges support from the Swiss National Science Foundation under grant Nr. 620-066162 during the early stages of this work.

2 In $b \rightarrow d \gamma$ decays, the $C_{7}^{(\prime)}$ are understood to be effective coefficients defined as in Eq. (3) with obvious flavour replacements and $\mathcal{H}_{\text {eff }}$ having further contributions from 4-quark operators $\propto V_{u b} V_{u d}^{*}$, see, e.g., Ref. [14] for details. 


\section{References}

[1] S. Hashimoto, M. Hazumi, J. Haba, J.W. Flanagan, Y. Ohnishi (Eds.), Letter of Intent for KEK Super B Factory, KEK Report 2004-4 (2004);

J. Hewett, D.G. Hitlin (Eds.), The Discovery Potential of a Super B Factory, Proceedings of the 2003 SLAC Workshops, SLAC-R-709 (2004), hep-ph/0503261.

[2] T. Inami, C.S. Lim, Prog. Theor. Phys. 65 (1981) 297;

T. Inami, C.S. Lim, Prog. Theor. Phys. 65 (1981) 1772, Erratum.

[3] See, for instance, T. Schietinger, Review of experimental results on rare radiative, semileptonic and leptonic $B$ decays, in: Proceedings of the XLIst Rencontres de Moriond (La Thuile, Italy, March 11-18, 2006), Eds. J.M. Frère, J. Trân Thanh Vân, G. Unal, (Thế Gió'i Publishers, Vietnam, 2006) p. 19, hep-ex/0605081.

[4] M. Gremm, F. Krüger, L.M. Sehgal, Phys. Lett. B 355 (1995) 579;

T. Mannel, S. Recksiegel, J. Phys. G: Nucl. Part. Phys. 24 (1998) 979.

[5] G. Hiller, A. Kagan, Phys. Rev. D 65 (2002) 074038.

[6] Z. Zhao, et al., Report of Snowmass 2001 Working Group E2: Electron-positron Colliders from the $\phi$ to the $Z$, in: Proceedings of the APS/DPF/DPB Summer Study on the Future of Particle Physics (Snowmass, USA, 2001), Ed. N. Graf, hep-ex/0201047.

[7] F. Legger, T. Schietinger, Phys. Lett. B 645 (2007) 204;

F. Legger, T. Schietinger, Phys. Lett. B 647 (2007) 527, Erratum.

[8] W.-M. Yao, et al., Particle Data Group, J. Phys. G: Nucl. Part. Phys. 33 (2006) 1.

[9] T. Mannel, G. A. Schuler, Phys. Lett. B 279 (1992) 194.

[10] A.F. Falk, M.E. Peskin, Phys. Rev. D 49 (1994) 3320.

[11] D. Buskulic, et al., ALEPH Collaboration, Phys. Lett. B 374 (1996) 319;

G. Abbiendi, et al., OPAL Collaboration, Phys. Lett. B 444 (1998) 539.

[12] J. Charles, A. Le Yaouanc, L. Oliver, O. Pène, J.C. Raynal, Phys. Rev. D 60 (1999) 014001.

[13] C.W. Bauer, S. Fleming, D. Pirjol, I. W. Stewart, Phys. Rev. D 63 (2001) 114020;

M. Beneke, A.P. Chapovsky, M. Diehl, T. Feldmann, Nucl. Phys. B 643 (2002) 431.

[14] See, for instance, G. Buchalla, A.J. Buras, M.E. Lautenbacher, Rev. Mod. Phys. 68 (1996) 1125.

[15] H.F. Jones, M.D. Scadron, Annals Phys. 81 (1973) 1. 
[16] S.W. Bosch, G. Buchalla, Nucl. Phys. B 621 (2002) 459.

[17] P. Ball, G.W. Jones, R. Zwicky, Phys. Rev. D 75 (2007) 054004.

[18] S.J. Brodsky, G.P. Lepage, Phys. Rev. D 24 (1981) 2848.

[19] G. Burdman, G. Hiller, Phys. Rev. D 63 (2001) 113008.

[20] R.J. Hill, T. Becher, S.J. Lee, M. Neubert, JHEP 0407 (2004) 081.

[21] R. Antunes Nobrega, et al., LHCb Collaboration, LHCb Reoptimized Detector Design and Performance Technical Design Report, CERN/LHCC 2003-030.

[22] W.G.D. Dharmaratna, G.R. Goldstein, Phys. Rev. D 53 (1996) 1073.

[23] J. Pursley, Talk presented on behalf of the CDF Collaboration at the 11th International Conference on B-Physics at Hadron Machines (Beauty 2006), 2529 September 2006, University of Oxford, UK, to be published in Nucl. Phys. B (Proc. Suppl.);

CDF Collaboration, CDF Public Note 8523, http://www-cdf.fnal.gov/physics/new/bottom/060921.blessed-sigmab/

[24] T.M. Yan, et al., Phys. Rev. D 46 (1992) 1148;

T.M. Yan, et al., Phys. Rev. D 55 (1997) 5851, Erratum;

J.G. Körner, M. Krämer, D. Pirjol, Prog. Part. Nucl. Phys. 33 (1994) 787.

[25] See, for instance, M. Anselmino, D. Boer, U. D'Alesio, F. Murgia, Phys. Rev. D 63 (2001) 054029.

[26] See, for instance, M. Smižanská, Nucl. Instr. and Meth. in Phys. Res. A 446 (2000) 138.

[27] See, for instance, G. Hiller, Phenomenology of New Physics, in: Proceedings of the Second International Conference on Flavor Physics and CP Violation, (Paris, France, June 3-6, 2003), eConf C030603, hep-ph/0308180.

[28] R.S. Chivukula, H. Georgi, Phys. Lett. B 188 (1987) 99; G. D’Ambrosio, G.F. Giudice, G. Isidori, A. Strumia, Nucl. Phys. B 645 (2002) 155.

[29] D. Atwood, M. Gronau, A. Soni, Phys. Rev. Lett. 79 (1997) 185.

[30] B. Grinstein, Y. Grossman, Z. Ligeti, D. Pirjol, Phys. Rev. D 71 (2005) 011504. 